\title{
配体调控镍催化烯烃区域发散性双碳官能团化反应
}

\author{
李晓芳储玲玲*
}

(东华大学化学化工与生物工程学院 先进低维材料研究中心 上海 201620)

\section{Ni-Catalyzed Ligand-Controlled Regiodivergent Reductive Dicarbofunctionalization of Alkenes}

\author{
Li, Xiaofang \\ Chu, Lingling*
}

(Center for Advanced Low-Dimension Materials, College of Chemistry, Chemical Engineering and Biotechnology,

Donghua University, Shanghai 201620)

吲哚酮、喹啉酮等两类重要杂环骨架，广泛存在于 具有重要生物活性的天然产物和药物分子中 ${ }^{[1]}$. 开发其 高效、高选择性的构建方法一直是合成化学领域中的研 究热点 ${ }^{[2]}$.

过渡金属催化含杂原子烯烃分子内环化/分子间交 叉偶联反应是构建杂环骨架的重要策略之一 ${ }^{[3]}$. 在该类 反应中, 金属物种与系链烯烃发生分子内迁移插入生成 烷基金属物种，再与另一偶联子发生交叉偶联. 尽管目 前已取得了重要进展, 但是如何抑制烷基金属物种的 $\beta-\mathrm{H}$ 消除途径仍面临挑战. 为了解决该难题, 一种常用 的策略就是采用 1,1-双取代的系链烯烃底物经由分子
内环化得到不含 $\beta-\mathrm{H}$ 的烷基金属物种，来高效构建含季 碳中心的杂环化合物.

近些年来，国内外不少课题组都在该领域做出了重 要贡献, 结合手性配体使用, 实现金属催化含季碳手性 中心的高效构建 ${ }^{[4-5]}$. 然而, 该策略大多得到动力学上更 有利的 5-exo 环化/交叉偶联产物, 而尚无 6-endo 环化/ 交叉偶联类型反应的报道. 这可能是由于在 6-endo 环化 过程中，烷基金属物种受空间位阻的影响难以进一步发 生交叉偶联，而更容易发生 $\beta-\mathrm{H}$ 消除或者质子化等过 程 ${ }^{[6]}$.

武汉大学高等研究院孔望清课题组 ${ }^{[7-9]}$ 致力于镍催<smiles>[R]c1ccc2c(c1)[C@]1(CCN(C)C1)C(=O)N2</smiles>

$\mathbf{R}=\mathbf{H}$, horsefiline $\mathbf{R}=\mathrm{OMe}$, amorolfine

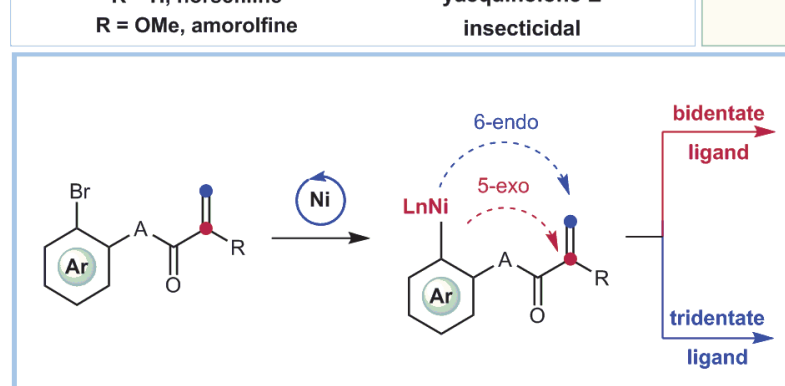

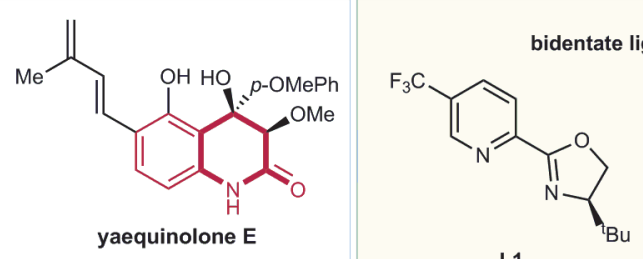

L1

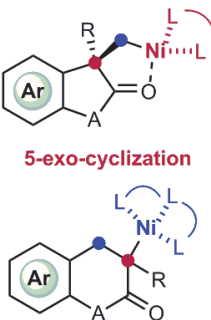

6-endo-cyclization tridentate ligand

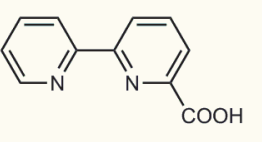

L3

图 1 配体调控烯烃的区域发散性双碳官能团化反应

Figure 1 Ligand-control regiodivergent alkene dicarbofunctionalization

* Corresponding author. E-mail: lingling.chu1@dhu.edu.cn. Published online September 8, 2021. 


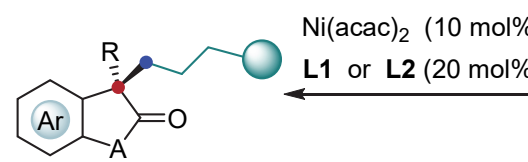

indolin-2-one

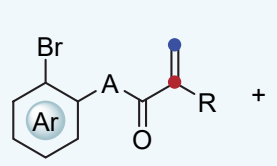

5-exo-selective

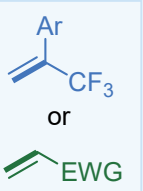

$\mathrm{NiBr}_{2} \cdot$ DME (10 mol\%)

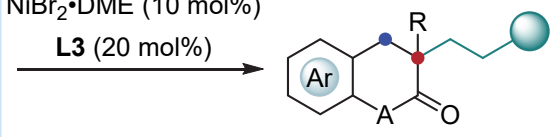

3,4-dihydroquinolin-2-one<smiles>[R7]c1cccc(C(CCCC2(C)C(=O)N(C)c3ccccc32)=C(F)F)c1</smiles>

$p-\mathrm{Cl}$, p-COMe, $81 \%, 96 \%$ ee $p-\mathrm{CN}$, $p-\mathrm{SO}_{2} \mathrm{Me}, \quad 48 \%, 95 \%$ ee $m-\mathrm{CO}_{2} \mathrm{Me}, 64 \%, 97 \%$ ee

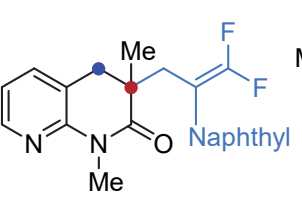
$89 \%$<smiles>Cc1cc2c3c(c1)C[C@](C)(CC(NO)=C(F)F)C(=O)N3CCC2</smiles>

$50 \%$

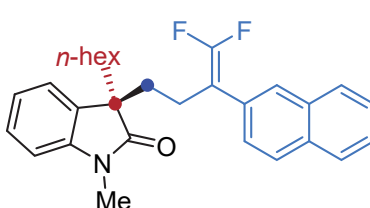

$43 \%, 93 \%$ ee

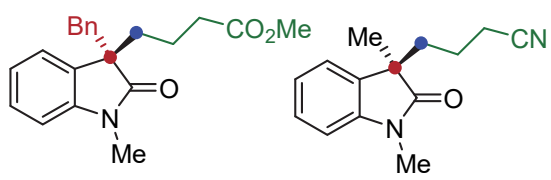

$75 \%, 93 \%$ ee
$66 \%, 92 \%$ ee 6-endo-selective

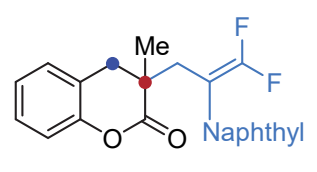
$49 \%$

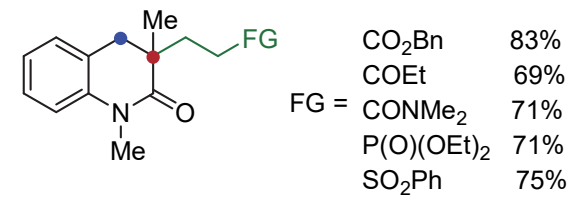

图 2 配体调控烯烃的发散性双官能团化反应底物拓展

Figure 2 Scope of ligand-controlled regiodivergent alkene dicarbofunctionalization

化烯烃、炔烃参与的环化偶联反应研究. 最近, 该课题 组发现, 利用配体调控活性镍物种镍中心周围的配位环 境, 可以实现系链烯烃参与的 5-exo 和 6-endo 分子内环 化/交叉偶联反应. 作者以含烯烃边链的芳基溴为模板 底物, 缺电子烯烃为偶联子, 锰粉为还原剂, 在镍催化 剂存在下对不同配体进行了篮选. 该作者发现，当使用 手性双齿配体 Pyrox (L1)或 Phox (L2)时, 能以优秀的区 域选择性、对映选择性得到 5-exo 环化/偶联的产物, 构 建含季碳手性中心的五元吲哚-2-酮结构单元; 而当使 用三齿配体 C6-羧基联吡啶 $(\mathbf{L} 3)$ 时, 能以优秀的区域选 择性得到 6-endo 环化/偶联的产物, 构建含季碳中心的 3,4-二氢喹啉-2-酮结构单元.

他们进一步对该配体调控 5-exo/6-endo 选择性环化/ 交叉偶联体系的普适性进行了考察. 在 5-exo 环化/偶联 反应中，芳基取代三氟甲基烯烃以及系链烯烃的取代芳 基溴均能高效参与反应, 以中等到优良的产率和高对映 选择性得到手性吲哚-2-酮产物. 在 6-endo 环化/偶联反 应中, 除含烯基酰胺边链的芳基溴底物外, 含烯基酯芳 基溴也能顺利参与环化反应，构建六元色满酮结构单 元. 该发散性体系均可进一步拓展到其他类型缺电性烯 烃, 如丙烯酸酯、丙烯腈和 $\alpha, \beta$-不饱和酮等, 构建相应 的烷基取代吲哚酮和喹啉酮结构单元. 为了进一步证明
该区域发散性体系的合成价值，他们基于 5-exo 和 6-endo 环化/烷基化偶联产物，进一步合成了各类具有 潜在生物活性的含氮杂环、并环等结构单元.

综上所述，武汉大学孔望清课题组发展了配体调控 的镍催化烯烃 5-exo 和 6-endo 分子内环化/交叉偶联反 应. 该方法为具有重要生物活性的含季碳中心五元、六 元杂环骨架的快速构建提供了高效、高选择性和发散性 的新合成方法.

\section{References}

[1] Prasat, K.; Chulabhorn, M.; Somsak, R. Curr. Top. Med. Chem. 2014, 14, 239.

[2] Pavithra, T.; Devi, E. S.; Maheswari, C. U. Asian J. Org. Chem. 2021, 10, 1861.

[3] Ping, Y.; Li, Y.; Zhu, J.; Kong, W. Angew. Chem., Int. Ed. 2019, 58, 1562.

[4] Tian, Z.-X.; Qiao, J.-B.; Xu, G.-L.; Pang, X.; Qi, L.; Ma, W.-Y.; Zhao, Z.-Z.; Duan, J.; Du, Y.-F.; Su, P.; Liu, X.-Y.; Shu, X.-Z. J. Am. Chem. Soc. 2019, 141, 7637.

[5] Jin, Y.; Wang, C. Angew. Chem., Int. Ed. 2019, 58, 6722.

[6] Parasram, M.; Iaroshenko, V. O.; Gevorgyan, V. J. Am. Chem. Soc. 2014, 136, 17926.

[7] Wang, K.; Ding, Z.; Zhou, Z.; Kong, W. J. Am. Chem. Soc. 2018 140,12364 .

[8] Ding, Z.; Wang, Y.; Liu, W.; Chen, Y.; Kong, W. J. Am. Chem. Soc. 2021, 143, 53 .

[9] Chen, J.; Wang, Y.; Ding, Z.; Kong, W. Nat. Commun. 2020, 11, 1882.

(Lu, Y.) 